\title{
Sexual selection and sexual conflict
}

\section{Candolin, Ulrika}

Elsevier

2019

Candolin , U 2019, Sexual selection and sexual conflict . in G Taft, M Nicholls \& M Willis (eds) , Encyclopedia of Ecology . 2 edn , vol. 1 , Elsevier , pp. 310-318 . https://doi.org/10.1016/B978-0-12-409548-9

http://hdl.handle.net/10138/304495

https://doi.org/10.1016/B978-0-12-409548-9.10886-3

acceptedVersion

Downloaded from Helda, University of Helsinki institutional repository.

This is an electronic reprint of the original article.

This reprint may differ from the original in pagination and typographic detail.

Please cite the original version. 


\title{
Sexual selection and sexual conflict
}

\author{
Ulrika Candolin \\ Dept of Biological \& Environmental Sciences \\ University of Helsinki, Finland
}

\begin{abstract}
Sexual selection is the cause of extravagant traits that confer no advantage in the struggle for survival, but improve mating and fertilization success. These traits are often highly exaggerated such as conspicuous ornaments and elaborate courtship displays. In this chapter I discuss the mechanisms of sexual selection - how and why sexually selected traits evolve - as well as the consequences that sexual selection can have at both the individual and the population level. Sexual conflict is an inevitable part of sexual selection, as the evolutionary interest of genetically different individuals always differ. This can result in the evolution of traits that severely harm females and thereby reduce population growth rate. Sexual selection can also influence speciation and hybridization, as well as the ability of species to adapt to rapid human-induced environmental changes. Sexual selection is currently a highly active research field, but many questions remain, such as the ultimate effect of sexual selection on population viability.
\end{abstract}

\section{Keywords}

alternative reproductive behaviors, courtship, environmental change, fertilization, Fisher's runaway process, good genes process, mate choice, mate competition, polygamy, reproductive success, sensory drive, sexual signal, sexually antagonistic co-evolution, sexually selected trait, sperm competition

\section{Glossary}

Effective population size, $\mathbf{N}_{\mathbf{e}}$ The number of individuals in a population that contribute offspring to the next generation.

Mating system Describes the manner in which males and females associate during mating in an attempt to maximize their lifetime reproductive success. Animal mating systems are categorized according to the number of mates a given sex is able to monopolize (monogamy, polygamy, and promiscuity). Such categorizations can be based on social bonds (social mating system) or on genetic paternity and maternity of the resultant offspring (genetic mating system).

Operational sex ratio, $\mathbf{0 S R}$ The average ratio of fertilizable females to sexually active males at any given time.

Potential reproductive rate, PRR The number of offspring that an individual can produce per unit time if unconstrained by mate availability. The rate is usually higher in males than females, as these produce many tiny sperm while females invest in larger ova and - more often than males in time consuming parental care.

Sexually antagonistic co-evolution, SAC A conflict between the evolutionary interests of the sexes that cause one sex to evolve traits that reduce the ability of the opposite sex to reproduce with other partners. The opposite sex can then evolve defense mechanisms against the manipulation, resulting in an 'arms race'. This can escalate and reduce female fecundity and, hence, population growth rate.

Transgenerational epigenetic effects Heritable alterations of gene expression without a change to the DNA sequence. Include DNA methylation, chromatin remodeling, histone modification, and non-coding RNA mechanisms. 


\section{Introduction}

Many species possess conspicuous traits that appear to confer no advantage in the struggle for survival, such as colorful plumes and feathers, melodic songs, loud calls, and exaggerated structures like horns and antlers. The selection pressure behind these traits is usually sexual selection; in the competition for mates and fertilizations, organisms have evolved a variety of traits that increase either their attractiveness to the opposite sex, or their success in the competition among rivals for access to mates and fertilizations. How these traits evolve and why has intrigued scientists for decades and grown into a vivid field of research. In particular, the question of why females prefer males with cumbersome traits, which obviously reduce the probability of survival - the peacock's train being a classic example - has been difficult to solve and subjected to much research.

The foundation of the theory of sexual selection was laid by Charles Darwin. He realized that the competition for mates can result in traits that are of no advantage in the struggle for survival, but evolve because of the advantage they convey over rivals in the competition for mates. Since then, the field of sexual selection has grown rapidly and provided detailed knowledge of how and why sexual selection operates. The consequences of sexual selection for populations, on the other hand, are still poorly known and in need of more research.

In this chapter, I go through the mechanisms behind sexual selection - how and why does sexual selection operate - as well as the consequences of sexual selection for populations. I discuss both the benefits and costs of sexual selection, as well as the question of whether sexual selection enhances or depresses population viability. An inevitable part of sexual selection is a conflict of interest between the sexes, as the evolutionary interest of genetically different individuals always differ. This conflict can result in the evolution of traits that are beneficial to the individual but detrimental to the population. It can, hence, play a fundamental role in determining the effect of sexual selection on populations.

\section{The mechanisms of sexual selection}

\subsection{Intra- and intersexual selection}

Sexual selection favors the evolution of traits that increase success in the competition for mates and fertilizations, ie, of sexually selected traits. These traits can vary from flamboyant ornaments that attract the opposite sex, such as the peacock's train, to weapons used to combat rivals, such as the antlers of deer.

Sexual selection operates through two main pathways, intra- and intersexual selection. Intrasexual selection occurs when competition among members of the same sex for mates and fertilizations favors the evolution of traits that provide an advantage in this competition, like large antlers in male deer that are used in male-male competition for access to females. Intersexual selection occurs when members of one sex choose mates of the other sex based on some traits. When the preferences for these traits are favored by selection, then the preferred traits may evolve and become exaggerated, like the train of the peacock (although strictly speaking intersexual selection is also competition within the sexes, but competition for being chosen and making the best choice). In most species, males are more competitive and females more choosy, but some degree of competitiveness and choosiness can occur in both sexes.

\subsection{Pre and postcopulatory sexual selection}

Sexual selection can be further divided into pre- and postcopulatory sexual selection. Precopulatory sexual selection takes place before mating and is the most obvious form of sexual selection. It was described already by Charles Darwin and was the focus of early sexual selection research. 
Postcopulatory sexual selection takes place after mating and received little attention until the publication of an influential paper by Geoff Parker in 1970 on sperm competition (Parker, 1970). Since then the field of postcopulatory sexual selection has grown rapidly and is today a highly active research field.

Precopulatory sexual selection can take many forms. It can be the competition for resources that are then used to attract mates - such as large territories - or the competition for direct access to mates such as physical fights over mates, or ritualized displays of dominance. Similarly, mate choice can take many forms. It can be based on preferences for particular traits in the opposite sex - such as colorful ornaments - or take the form of resistance against mating attempts. The latter is common in species where the potential reproductive rate of males is much higher than that of females and males therefore attempt to copulate with unwilling females. A high potential reproductive rate reduces the cost of each mating attempt, which makes males less choosy.

Postcopulatory sexual selection includes sperm competition and cryptic female choice (Birkhead and Pizzari, 2002). Sperm competition arises when many males mate with a female, either because the female chooses to mate with multiple males or because multiple males overcome female resistance to mate (male harassment). It can favor the evolution of male traits that increase fertilization success, such as mate guarding, copulatory plugs that prevent female re-mating, or seminal proteins that influence female physiology. For instance, males of the fruit fly (Drosophila melanogaster) inseminate females with sperm that are embedded in a cocktail of seminal substances, which stimulate ovulation, deactivate sperm that are already stored in the reproductive tract of the female, and act as an anti-aphrodisiac, discouraging females from participating in future copulations (Chapman, 2001).

Cryptic female choice occurs when females have some control over which males' sperm fertilize the ova. It is 'cryptic' in the sense that the choice takes place hidden within the reproductive tract of the female. Cryptic female choice is less investigated than sperm competition, and its prevalence is poorly known. An indication of the occurrence of cryptic female choice is complex female sperm storage organs that rapidly co-evolve with sperm and ejaculate traits. Cryptic choice has been documented in species such as fruit flies, stalk-eyed flies and feral fowls. Female feral fowls (Gallus gallus domesticus), for instance, have organs that can expel the ejaculates of lower-ranking males and, in so doing, increase the insemination success of higher ranking males (Pizzari and Birkhead, 2000) (Figure 1).

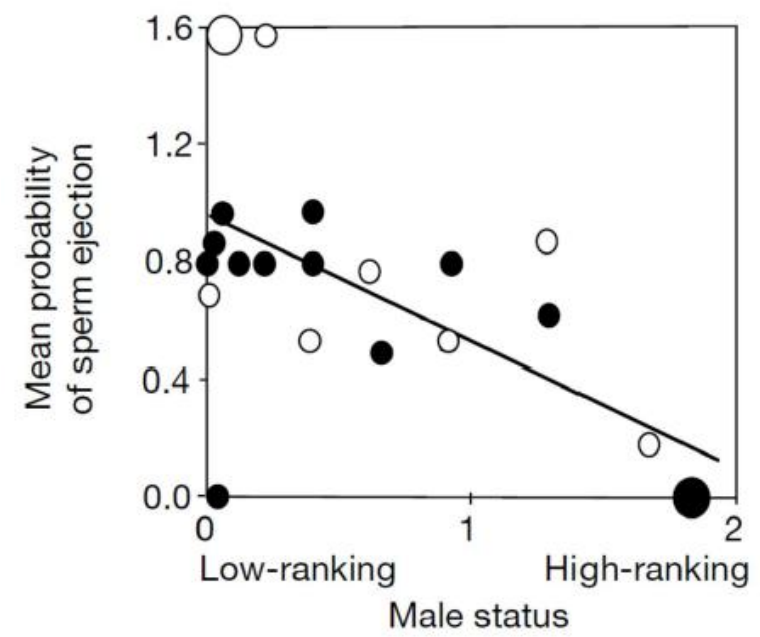

Figure 1. An example of cryptic female choice; to increase the probability of ova being fertilized by sperm of high quality males, females feral fowls eject the sperm of low ranking males. Small data points represent one male, large data points, two males. Different colors refer to different years. Small data points represent one male, large datapoints, two males. Reproduced from Pizzari, T., \& Birkhead, T. R. (2000). Female feral fowl eject sperm of subdominant males. Nature 405: 787-789. 


\subsection{The strength of sexual selection}

The strength of sexual selection on traits used in mate competition and mate choice depends on the fitness costs and benefits of these traits. A common cost that constrains sexual selection is the expenditure of a limited pool of resources on competition and choice, as this reduces the availability of resources for other fitness enhancing traits. For instance, the allocation of time, energy and nutrients to mate competition and mate choice can reduce the amount available for feeding and growth. Other common costs are increased risk of predation and parasite infections. Mate searching, in particular, that increases activity can raise encounter rate with predators or parasites, while ornaments and courtship behaviors can make individuals more conspicuous to these. An example is threespine stickleback males, who use a conspicuous red nuptial coloration to attract females and repel competing males, which increases their risk of predation from piscivorous fishes (Johnson and Candolin, 2017) (Figure 2).
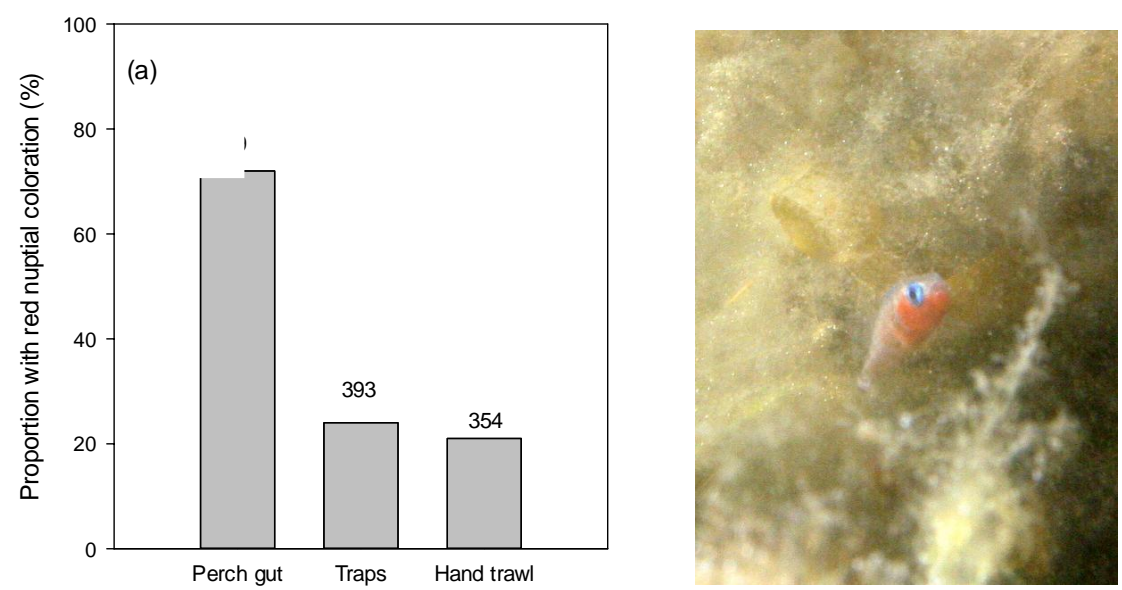

Figure 2. The conspicuous red nuptial coloration of threespine stickleback males increases their risk predation risk. The proportion of red colored sticklebacks that are caught by a predator, the European perch (Perca fluviatilis), is higher than the proportion in the population. Reproduced from Johnson, S., \& Candolin, U. (2017). Predation cost of a sexual signal in the threespine stickleback. Behavioral Ecology, 28: 1160-1165.

The benefit of competition and mate choice, which favors the evolution of sexually selected traits, is improved fertilization success in relation to other individuals in the population. This benefit has to be larger than the costs, ie, it has to increase lifetime fitness in terms of number of copies of the individual's genes that are transferred to subsequent generations. Relative mating success - not absolute mating success - is central, as relative success influences the representation of the genes of the individual in the next generation.

These costs and benefits of sexually selected traits depend in turn on a range of factors, both environmental and intrinsic to the individual. These are variation among individuals in the fitness benefits they can offer (if the variation is small, the benefit of mate choice is also small); mate encounter rate, which determines the possibility of choice; the operational sex ratio, which influences the strength of competition for mates; and life-history traits, such as number of lifetime reproductive opportunities, which in turn are determined by a range of factors, such as body condition, parental effort and age. For instance, the white plumage patches of collared flycatchers (Ficedula albicollis) show positive age-dependent expression, such that the size of the ornaments increases as individuals age (Evans, Gustafsson, and Sheldon, 2011) (Figure 3). This increase in size could be a consequence of the cost of ornament expression decreasing when future reproductive 
opportunities decline towards the end of life, as this could allow individuals to increase their investment into the sexually selected trait as a terminal investment.
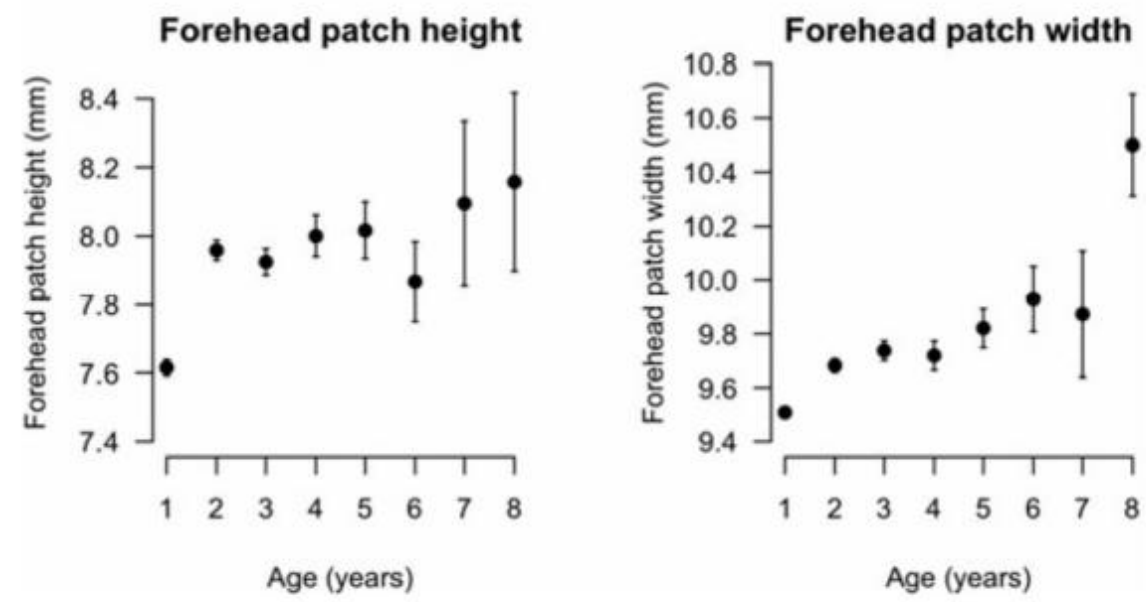

Figure 3. The expression of a sexually selected trait, the white forehead patch size of the collared flycatcher, increases with age. This could be a consequence of reduced costs of expressing the trait in terms of loss of reproductive opportunities towards the end of the life, as reproductive opportunities then decrease. Reproduced from Evans, S. R., Gustafsson, L., \& Sheldon, B. C. (2011). Divergent patterns of age-dependence in ornamental and reproductive traits in the collared flycatcher. Evolution 65: 1623-1636.

\subsection{Sexual conflict}

A conflict of interest always arises when genetically different individuals attempt to maximize their fitness. This is because fitness is a relative measure, and the proportion of genes passed on to the next generation can only increase if that of other individuals decrease. In a mating context, a conflict of interest arises when the sexes have conflicting optimal fitness strategies (Arnqvist and Rowe, 2005). For instance, females may benefit if males increase their parenting effort, as this saves resources for investment into fecundity and future reproductive opportunities. M ales, on the other hand, may benefit from leaving parental care to females, as this frees up time and energy for searching for new mates, to gain additional offspring. An example is males of the European starling (Sturnus vulgaris), who decrease their paternal effort when more nestboxes are available and the opportunity to attract additional mates is high (Smith, 1995).

Sexual conflict can be of two types: interlocus and intralocus. Interlocus sexual conflict occurs when one sex evolves traits that enhance its reproductive success at the expense of the fitness of its mating partners. The other sex may then evolve counteradaptations, which are determined by other genes (loci), which leads to antagonistic coevolution between the sexes. For example, male fruit flies produce seminal proteins that reduce the probability that females will reproduce with other males. This has in turn induced females to evolve counteradaptations to resist their impact. Such sexually antagonistic coevolution can result in bizarre traits, such as the spines on the genitalia of male seed beetles, which harm females (Ronn, Katvala, and Arnqvist, 2007) (Figure 4). Other examples of traits that evolve through interlocus sexual conflict are mate guarding, physical harassment, and resistance against mating attempts.

Intralocus sexual conflict occurs when the sexes have different optima for a trait that is expressed in both sexes and determined by the same genes, such as horn size or body coloration.

The conflict of interest is between individuals, between the male and the female attempting to reproduce. At the population level, the fitness of the sexes is equal when the sex ratio is equal, as each offspring belongs to both sexes. Thus, the variation in mating success is among individuals 
within each sex competing for mates. The variation is usually higher in males, as these typically have a higher potential reproductive rate, which, hence, are more competitive.

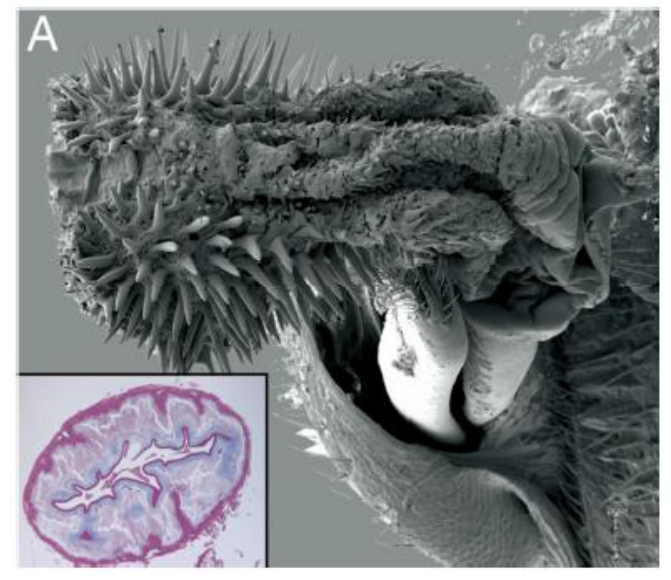

Figure 4. Male seed beetles (Coleoptera bruchidae) have evolved spines on their genitalia that enhance stability during copulation, but cause harm to females. Females have in turn evolved resistance to these spines, through tougher copulatory ducts, which has led to sexually antagonistic coevolution (SAC) with males evolving more and more spines on their genitalia. Reproduced from Ronn, J., Katvala, M., \& Arnqvist, G. (2007). Coevolution between harmful male genitalia and female resistance in seed beetles. Proceedings of the National Academy of Sciences of the United States of America 104: 10921-10925.

\section{The evolution of sexually selected traits}

Whether sexual selection results in the evolution of sexually selected traits depends on the strength of sexual selection, as well as on the heritability of the traits. The strength of sexual selection depends on the costs and benefits of sexually selected traits, as was discussed earlier in this chapter. The heritability of sexually selected traits depends in turn on the magnitude of variation among individuals in the traits, and the degree to which this variation is determined by genetic (ie, allelic) and nongenetic mechanisms. If the variation among individuals in traits is genetically determined and in the direction of selection, then the traits may evolve through genetic changes. If, on the other hand, the variation is nongenetic and caused by phenotypic plasticity and environmental effects, then the traits might still evolve but through transgenerational effects, such as parental effects.

An increasing number of studies find nongenetic inheritance to contribute to evolution. An example is the loudness of an acoustic signal in a grasshopper (Chorthippus biguttulus), which is determinde by the diet of the parents and, hence, inherited through parental effects (Franzke and Reinhold, 2013). The prevalence of nongenetic inheritance, and the persistence of the effects, are, however, poorly known. Transgenerational epigenetic effects (such as the methylation of DNA) might persists across many generations, as these alter gene expression, but how common epigenetic effects are in a sexual selection context, and for how many generations they can persist, are unknown.

Genetic and nongenetic inheritance may interact in influencing the evolution of sexually selected traits. This can complicate the prediction of evolutionary responses to sexual selection. Nongenetic inheritance may accelerate evolution by increasing the amount of heritable phenotypic variation that selection can act on, and by causing rapid changes in trait expression, as these may persist only from one generation to the next.

The heritability of sexually selected traits - whether genetic or nongenetic - is dependent on the magnitude of variation among individuals in the traits, as well as on correlations among the traits. Low variation and negative correlations can constrain evolution. For instance, in Drosophila serrata, female choice is based on male cuticular hydrocarbons (CHCs), but in populations with pleiotropic covariation among the traits, selection on several traits result in no evolutionary response (Hine, 
McGuigan, and Blows, 2014). This is probably because covariation among the traits reduces genetic variation in trait combinations in the direction of selection.

\section{The theory of sexual selection}

The evolution of traits used in male-male competition for access to females is easily understood. A male that wins over other males in physical fights, or acquires a larger territory than other males, gains access to more females and have a higher mating success. The traits that ensures or correlates with this success, such as large body size or antlers, are then favored by sexual selection.

A more challenging question is the evolution of female mate preferences for traits that are harmful to survival, such as colorful ornaments that attract predators, or vigorous courtship displays that are energetically costly. This question occupied scientists during the early years of sexual selection research, and today we have a fairly good understanding of the factors that promote the evolution of female (or male) mate preferences. These mechanisms can be divided into two main groups; direct and indirect benefits of mate choice.

The direct benefits hypothesis states that females evolve preferences for traits that reflect some direct benefits of mating with the male, such as male parenting ability or fertilization success. By basing their mate choice on these traits, females improve their own reproductive success in terms of number of offspring produced. For example, females of the northern cardinal (Cardinalis cardinalis) use the brightness of the males' plumage to gain information on the proportion of the offspring feeding that the males would provide (Linville and Breitwisch, 1997) (Figure 5).

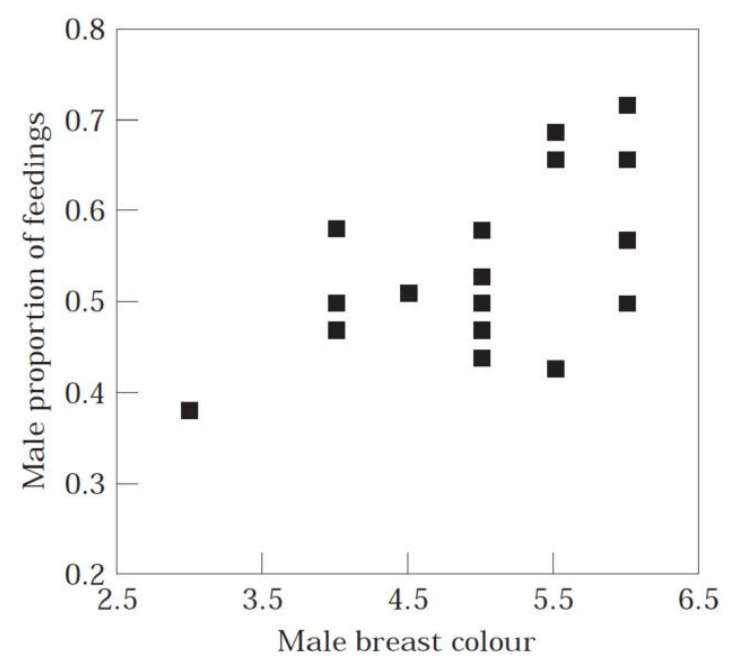

Figure 5. The breast color of male northern cardinals reflects the proportion of the offspring feeding that the male will provide. Thus, the color can be used as an indicator of the direct benefit that the female would gain by mating with the male. Reproduced from Linville, S. U., \& Breitwisch, R. (1997). Carotenoid availability and plumage coloration in wild populations of northern cardinals. Auk 114: 796-800.

The indirect benefits hypothesis states that females evolve preferences for traits that reflect genetic benefits and, hence, improve offspring fitness, and thereby indirectly the fitness of the female. These models can be further divided into two groups: 1) good genes models that assume that offspring inherit genes that improve their survival, and 2) Fisherian runaway process that assumes that offspring inherit the attractiveness of their father and, hence, have a high mating success.

The Fisherian process includes self-reinforcing coevolution between the trait and the preference. $M$ ales evolve a trait that is preferred by females, and the trait and the preference then become genetically coupled. When the female preference increases in frequency in the population, exaggeration of the male trait yields an increasing advantage to the male and the trait evolves further, until the 'runaway' evolution is stopped by opposing natural selection, ie, by the costs of the 
trait. The degree to which Fisher's runaway process occurs in nature is poorly known, but it could commonly operate alongside other processes that drive the evolution of sexually selected traits.

Other mechanisms that can influence the evolution of mate preferences are the sensory drive mechanism and sexually antagonistic coevolution. The sensory drive model states that the presence of pre-existing sensory biases influence which traits females (or males) pay attention to and, hence, which traits will become exaggerated. For instance, females of a tropical fish - the swordtail characin (Corynopoma riisei) - feed on ants, which has favored the evolution of male ornaments with the shape of an ant (Kolm, Amcoff, M ann, and Arnqvist, 2012). Although sensory drive can initiate the evolution of sexually selected traits and preferences, it is unlikely to drive the evolution alone. It needs to be coupled with direct or indirect benefits of choice for the sexually selected traits to persist in the population.

The sexually antagonistic coevolution model builds on the conflict of interest between the sexes, as discussed earlier. The sexes are caught in a perpetual sexual arms race where one sex attempts to overcome the resistance of the other sex, which then evolves stronger resistance, until the benefit of further escalation is outweighed by the cost. Antagonistic coevolution is assumed to operate alongside other processes and contribute to the evolution of sexually selected traits, but evidence for this is so far limited.

\section{The maintenance of honesty}

An intriguing question is how honest sexual signals of mate quality are ensured - ie, what prevents individuals from deceptively increasing their signaling effort in order to attract more mates. A solution has been posed by the handicap principle; it postulates that sexually selected signals have to be costly so that poor quality individuals cannot express as costly signals as good quality individuals. This is analogous to the 'big house and big cars' correlation, where individuals with much resources can invest in both, in the case of sexually selected signals, in both signals and other fitness enhancing traits. One mechanism that can ensure a positive correlation between viability and the expression of signals is condition-dependence of signals. For instance, the tail length of barn swallows (Hirundo rustica) indicates viability because long tails are costly in terms of energy and nutrient demands and, hence, only individuals in prime condition - who have a high viability - can sustain the cost (Møller, 1994).

\section{Relation to mating systems}

The strength of sexual selection is closely related to the mating system. In monogamous mating systems the number of mating opportunities is limited and the competition for mates is consequently less strong. Sexual selection is then weak and highly exaggerated traits are unlikely to evolve. In polygamous or promiscuous mating systems, on the other hand, competition for mates is stronger and exaggerated sexually selected traits are more likely to evolve. Exaggeration of male traits is particularly likely, as males usually have a higher potential mating rate than females. Male California sea lions (Zalophus californianus), for instance, can gather harems of 10-20 females on their territories, which results in fierce fighting among males for females. Such intense competition increases the strength of sexual selection on traits that improve success in male-male competition, such as large male size. Consequently, profound sexual size dimorphism has evolved, with males being three to four times heavier than females.

The mating strategy an animal adopts depends on the factors that determine the number of mates an individual can monopolize and fertilize, ie, the mating skew. These factors can be demographic, social and ecological. For instance, the abundance and distributions of food can influence the number of females a male can provide for, and, hence, the intensity of competition for mates and fertilizations. Similarly, the need for male parental care can restrict the number of females a male can mate with and thereby the strength of sexual selection. 


\section{Alternative mating behaviors}

When the competition for mates is intense, sexual selection may cause the evolution of alternative mating tactics. An individual with a low probability of gaining matings through the dominant mating tactic - such as fights or courtship - may adopt an alternative mating tactic, such as sneaking or forced copulations. For example, male guppies (Poecilia reticulata) that fail to gain matings through courtship may attempt forced copulations.

Such alternative tactics can be adopted throughout life, if the fertilizations success through them is higher than the success through the dominant tactic. For instance, males who are born at an unfavorable time of the year, and therefore suffer from small size throughout life, may remain as small sneaker males. On the other hand, if the males can grow in size with time, they may switch to the dominant tactic when reaching a critical size. If the lifetime fitness benefit of the two tactics is equal, then the tactics may become genetically fixed and persist throughout life, as both are then evolutionary stable.

Sexual selection can favor the adoption of an alternative tactic alongside the dominant tactic as a strategy to maximize fitness. For instance, stickleback males that have established a territory and successfully attracted females through courtship may, when given a chance, also capitalize on the courtship effort of neighboring males and sneak fertilize the eggs females lay in their nests (Candolin and Vlieger, 2013). Similarly, both males and females, of a range of species, engage in extra pair copulations in the pursuit of higher lifetime fitness. Sexual selection can then favor the evolution of traits that increases the success of such behaviors, such as a rapid switch to dull coloration when attempting to gain sneak fertilizations.

\section{Population consequences}

The consequences that sexual selection has at the population level is debated. This is because sexual selection can both enhance and depress population viability, and whichever dominates depends on the characteristics of the species and the environmental conditions.

The good genes process predicts that populations benefit from sexual selection; individuals of high genetic quality - who are well adapted to prevailing conditions - are expected to have the highest mating success, which ensures that the population consists of individuals well suited to the environment. Similarly, the model of direct benefits of mate choice - according to which females enhance their lifetime reproductive success by being choosy - predicts that sexual selection can improve population viability as more offspring are born into the population.

The Fisherian runaway process, on the other hand, can result in reduced population viability. This is because the extravagant traits and expensive choice behaviors favored by the process can use up resources needed for population growth, or reduce survival by increasing predation or parasite infection risks. Similarly, the cost of sexual selection that ensures honest signaling of mate quality in both the good genes and the direct benefit models can reduce resources available for population growth, as well as increase mortality risk. An even more serious scenario arises when sexual conflict results in males evolving traits that harm females. These can drastically reduce female fecundity and, hence, population growth rate.

The degree to which the costs and benefits of sexually selected traits influence population viability depends on a range factors. In particular, any factor that alters female fecundity may alter population growth rate, as growth depends crucially on female fecundity. However, if only males suffer increased mortality because of the costs of sexually selected traits, and the surviving males can fertilize all females in the population, then the reproductive rate of the population need not decrease. On the contrary, increased male mortality could improve population growth rate if more resources are left to females to invest in fecundity. Similarly, if the use of resources in male-male competition has no influence on female fecundity, or the growth and survival of the offspring, then 
the cost of sexual selection may not reduce population growth rate. For instance, if males consume other food items, or inhabit different areas than the females and the offspring, then their use of resources may not influence population growth rate.

Antagonistic coevolution between the sexes, which results in males harming females and reducing their fecundity, can severely depress population viability. Little is known, however, about such effects in nature. Populations that suffer heavily from sexual conflict and male harm are not expected to be long-lived, as selection at the population level is expected to wipe out poorly performing populations. The frequency of harmful male traits could consequently be low in nature.

In general, the ultimate effect of sexual selection on population viability is poorly known. Sexual selection can be a strong force in driving the evolution of traits, but its influence on population viability, and the factors that determine its impact, need more investigations.

\section{Sexual selection and speciation}

While the influence of sexual selection on population viability and extinction risk is poorly known, its impact on hybridization and speciation is better understood. Sexual selection can be an important player in species divergence and convergence, given its power to drive changes in mate recognition traits. For example, the evolution of reproductive isolation between two frog species - Pseudacris feriarum and $P$. nigrita - is driven by sexual selection; by reinforcing differences in their acoustic signals and preferences - through reproductive character displacement - sexual selection acts against hybrids and promotes divergence (Lemmon and Lemmon, 2010).

Sexual selection may frequently interact with natural selection in driving species divergence or convergence. An example is sympatric species pairs of stickleback, which have diverged in parallel in both sexual and ecological trait in many lakes. Species that inhabit the benthic zone of lakes have evolved to base their mate choice mainly on body size, while species that inhabit the limnetic zone mainly use male nuptial coloration as a mate choice cue (Boughman, Rundle, and Schluter, 2005)

\section{Sexual selection in changing environments}

Environments are changing at an accelerating rate because of human activities. Whether species will be able to adjust to these changes can be influenced by sexual selection. Sexual selection can either facilitate or hinder adaptation to environmental change, depending on how it influences the number and quality of individuals reproducing in a population (Candolin and Heuschele, 2008).

If the change in the environment increases the costs of sexually selected traits, or, alternatively, decreases their benefits, then the survival or reproductive success of individuals may decrease. For instance, a shortage of food may increase the relative energetic cost of courtship activity, which can reduce survival, while a change in visibility may reduce the ability of females to evaluate ornamental traits and, hence, result in maladaptive matings. For example, female threespine stickleback that spawn in water that has become more turbid because of anthropogenic eutrophication are less able to evaluate the visual cues of males, such as their ornaments and courtship activity. This results in females increasingly spawning with males siring offspring with low survival probability (Candolin, Tukiainen, and Bertell, 2016) (Figure 6).

Correspondingly, if the costs of sexually selected traits decrease or their benefits increase in the altered environment, then sexual selection may improve the viability of the population. For instance, an increased variation among individuals in viability because of harsher conditions could increase the benefit of careful mate choice, which in turn could ensure that the individuals best adapted to the novel conditions have the highest reproductive success and, hence, accelerate adaptation. Moreover, if sexual selection had a negative effect on the population in the past environment, because of costs of sexually selected traits, then a relaxation of sexual selection in the altered environment could benefit the population. For instance, if antagonistic sexual selection reduced 
population growth rate in the past environment, a reduced ability of one of the sexes to manipulate the other sex in the altered environment could improve the growth rate of the population.

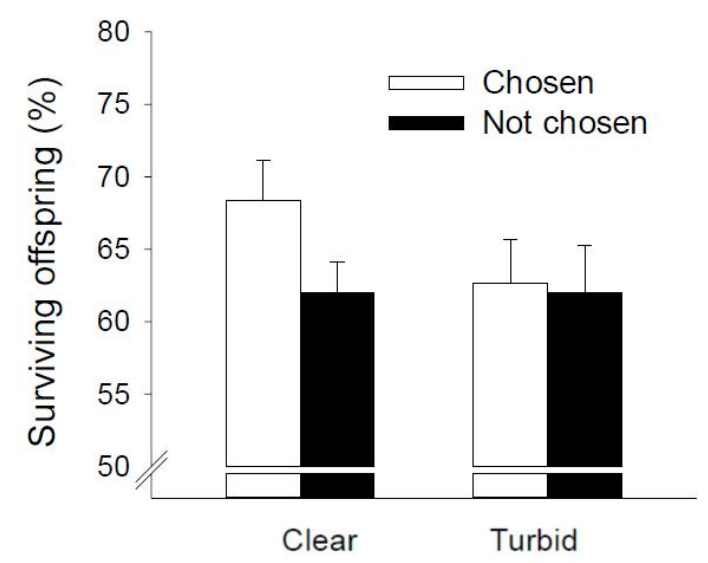

Figure 6. Threespine stickleback females choosing between two courting males more often chose the male that sired offspring with low survival probability when the choice was made in turbid water rather than in clear water. Anthropogenic eutrophication can consequently cause maladaptive mate choices in the stickleback. Reproduced from Candolin, U., Tukiainen, I., \& Bertell, E. (2016). Environmental change disrupts communication and sexual selection in a stickleback population. Ecology 97: 969-979.

Sexual selection usually increases the mating skew, which decreases effective population size. This could increase the risk of extinction, as small populations have a higher probability of extinction than larger populations, because of higher demographic stochasticity and lower rate of adaptation. In support of this, populations introduced to island - which often involve only a few individuals - are less likely to establish themselves if sexual selection is strong rather than weak (Sorci, M øller, and Clobert, 1998).

A factor that could limit the cost of sexually selected traits in altered environments, and prevent negative effects on the population, is condition-dependence of sexually selected traits. If individuals develop less exaggerated traits when conditions become harsher, then the cost could decrease and improve population viability. Currently, little is known about the impact that sexual selection has on the ability of species to adjust to rapid human-induced changes. It is clear, however, that sexual selection could play a major role given its influence on the reproductive success of individuals and their fitness.

\section{References}

Arnqvist, G., and Rowe, L. (2005). Sexual Conflict. Princeton, NJ.: Princeton University Press.

Birkhead, T. R., and Pizzari, T. (2002). Postcopulatory sexual selection. Nature Reviews Genetics 3, 262-273.

Boughman, J. W., Rundle, H. D., and Schluter, D. (2005). Parallel evolution of sexual isolation in sticklebacks. Evolution 59, 361-373.

Candolin, U., and Heuschele, J. (2008). Is sexual selection beneficial during adaptation to environmental change? Trends in Ecology and Evolution 23, 446-452.

Candolin, U., Tukiainen, I., and Bertell, E. (2016). Environmental change disrupts communication and sexual selection in a stickleback population. Ecology 97, 969-979.

Candolin, U., and Vlieger, L. (2013). Should attractive males sneak: the trade-off between current and future offspring. PLoS ONE, 8(3): e57992.

Chapman, T. (2001). Seminal fluid-mediated fitness traits in Drosophila. Heredity 87, 511-521.

Evans, S. R., Gustafsson, L., and Sheldon, B. C. (2011). Divergent patterns of age-dependence in ornamental and reproductive traits in the collared flycatcher. Evolution 65, 1623-1636. 
Franzke, A., and Reinhold, K. (2013). Transgenerational effects of diet environment on life-history and acoustic signals of a grasshopper. Behavioral Ecology 24, 734-739.

Hine, E., M cGuigan, K., and Blows, M. W. (2014). Evolutionary Constraints in High-Dimensional Trait Sets. American Naturalist 184, 119-131.

Johnson, S., and Candolin, U. (2017). Predation cost of a sexual signal in the threespine stickleback. Behavioral Ecology 28, 1160-1165.

Kolm, N., Amcoff, M., Mann, R. P., and Arnqvist, G. (2012). Diversification of a Food-M imicking Male Ornament via Sensory Drive. Current Biology 22, 1440-1443.

Lemmon, E. M., and Lemmon, A. R. (2010). Reinforcement in chorus frogs: lifetime fitness estimates including intrinsic natural selection and sexual selection against hybrids. Evolution 64, 17481761.

Linville, S. U., and Breitwisch, R. (1997). Carotenoid availability and plumage coloration in wild populations of northern cardinals. Auk, 114, 796-800.

Møller, A. P. (1994). Sexual Selection and the Barn Swallow. Oxford: Oxford University Press.

Parker, G. A. (1970). Sperm competition and its evolutionary consequences in insects. Biological Reviews of the Cambridge Philosophical Society 45, 525-567.

Pizzari, T., and Birkhead, T. R. (2000). Female feral fowl eject sperm of subdominant males. Nature 405, 787-789.

Ronn, J., Katvala, M., and Arnqvist, G. (2007). Coevolution between harmful male genitalia and female resistance in seed beetles. Proceedings of the National Academy of Sciences of the United States of America 104, 10921-10925.

Smith, H. G. (1995). Experimental demonstration of a trade-off between mate attraction and paternal care. Proceedings of The Royal Society of London Series B-Biological Sciences 260, 45-51.

Sorci, G., Møller, A. P., and Clobert, J. (1998). Plumage dichromatism of birds predicts introduction success in New Zealand. Journal of Animal Ecology 67, 263-269.

\section{Further reading}

Andersson M (1994) Sexual selection. Princeton: Princeton: University Press.

Andersson, M., and Simmons, L. W. (2006). Sexual selection and mate choice. Trends in Ecology and Evolution 21, 296-302.

Birkhead, T. R. and Møller, A. P. (1998). Sperm Competition and Sexual Selection. San Diego, CA:

Academic Press

Chenoweth, S. F., and M cGuigan, K. (2010). The Genetic Basis of Sexually Selected Variation. Annual Review of Ecology, Evolution, and Systematics 41, 81-101.

Eberhard, W. G. (2009). Postcopulatory sexual selection: Darwin's omission and its consequences. Proceedings of the National Academy of Sciences of the United States of America 106, 10025-10032. Emlen, D. J. (2008). The Evolution of Animal Weapons. Annual Review of Ecology, Evolution, and Systematics 39, 387-413.

Hosken, D. J. and House, C. M. (January 2011). Sexual Selection. Current Biology 21 R62-R65. Jones, A. G., and Ratterman, N. L. (2009). Mate choice and sexual selection: What have we learned since Darwin? Proceedings of the National Academy of Sciences of the United States of America 106, 10001-10008.

Kokko, H., Jennions, M. D., and Brooks, R. (2006). Unifying and testing models of sexual selection. Annual Review of Ecology, Evolution, and Systematics 37, 43-66.

Mousseau, T. A., and Fox, C. W. (1998). The adaptive significance of maternal effects. Trends in Ecology and Evolution, 13, 403-40.

Ritchie, M. G. (2007). Sexual selection and speciation. Annual Review of Ecology, Evolution, and Systematics 38, 79-102.

Servedio, M. R. and Boughman, J. W. (2017). The Role of Sexual Selection in Local Adaptation and Speciation Annual Review of Ecology, Evolution, and Systematics 48, 85-109. 
Zahavi, A. (1975). M ate selection: a selection for a handicap. Journal of Theoretical Biology 53, 205214. 